\section{De Fontaine Selected for Turnbull Lectureship}

A distinguished scientist who has long been a leader in research and education in materials science has been selected to deliver the 1995 David Turnbull Lecture. Didier de Fontaine, professor of materials science and engineering at the University of California at Berkeley, was cited by the selection committee "in recognition of fundamental contributions and insights in the fields of order/disorder phenomena in materials and computational techniques for phase diagrams."

The David Turnbull Lectureship recogrizes the career of a scientist who has made outstanding contributions to understanding materials phenomena and properties through research, writing, and lecturing, as exemplified by David Turnbull.

Through the efforts of de Fontaine, the nature and origin of ordering reactions in a wide variety of materials have been clarified, and the insight generated from this work has been crucial to the interpretation of experimental information. The many citations to his earlier work on the $\Omega$-phase and recent work on oxygen ordering in $\mathrm{YBa}_{2} \mathrm{Cu}_{3} \mathrm{O}_{7-\delta}$ testify to the relevance of his work for the materials community at large.

De Fontaine has made valuable contributions to the techniques used for the computation of phase diagrams. By applying Kikuchi's Cluster Variation Method to the computation of superstructure phase diagrams, he was one of the first to show that the correct topology of these diagrams was determined by configurational entropy effects and could therefore not be obtained with simple mean field theory.

Along with one of his first students, Juan Sanchez, he clarified the nature of the interactions in lattice models used for

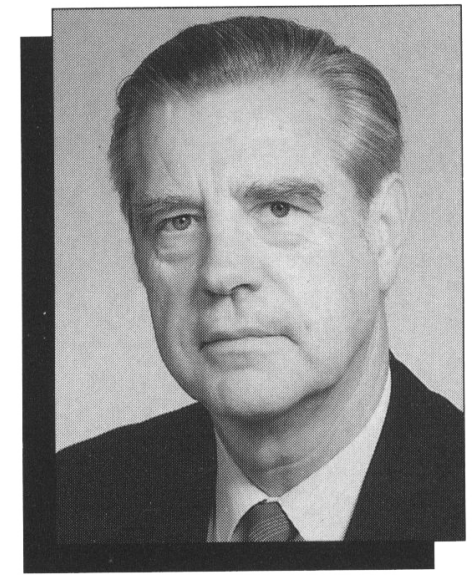

the computation of phase diagrams to show that they are not atomic potentials, but rather effective cluster interactions that can be derived from first-principles calculations through a cluster expansion of the energy.

De Fontaine's recent research is on phase transformations in alloys, crystallography, and thermodynamics of phase changes, particularly ordering reactions and phase separation. He used high-voltage and high-resolution electron microscopy to study long-period superstructures in ordered alloys, and performed calculations of phase diagrams by combined electronic band structure and cluster variation techniques.

De Fontaine received his MS degree in metallurgical engineering from the University of Louvain in Belgium in 1955, and his PhD degree in materials science from Northwestern University in 1967. $\mathrm{He}$ began his distinguished teaching career as a lecturer in 1962 at Lovanium University in the Congo, then conducted postdoctoral research at Bell Telephone Laboratories from 1966 to 1968.

He joined the University of California at Los Angeles in 1969, where he remained for nearly a decade. Following six months as a visiting professor at the École Polytechnique de Grenoble, he joined the University of California at Berkeley in 1979. He is currently professor of materials science at Berkeley and associate faculty scientist at the Lawrence Berkeley Laboratory.

De Fontaine has twice received an award for Outstanding Scientific Accomplishment in Metallurgy and Ceramics from the U.S. Department of Energy, as well as two Exceptional Creativity Awards from the National Science Foundation. He is a fellow of both the Japan Society for the Promotion of Science and the American Physical Society. Among his more than 200 publications in the fields of materials science and condensed matter physics are two invited review articles for Solid State Physics, the second of which appeared last year in a special volume dedicated to editor emeritus David Turnbull.

De Fontaine will present the Turnbull Lecture on Monday, November 27, at 10:15 a.m. in Symposium P: Materials Theory, Simulations, and Parallel Algorithms, Essex South, Westin Hotel. The title of his talk is "From Gibbsian Thermodynamics to Electronic Structure: Non-Empirical Study of Alloy Phase Equilibria."

Previous recipients of the David Turnbull Lectureship are Thomas R. Anthony, General Electric Company; Morris Cohen, Massachusetts Institute of Technology; and Arthur S. Nowick, Columbia University.

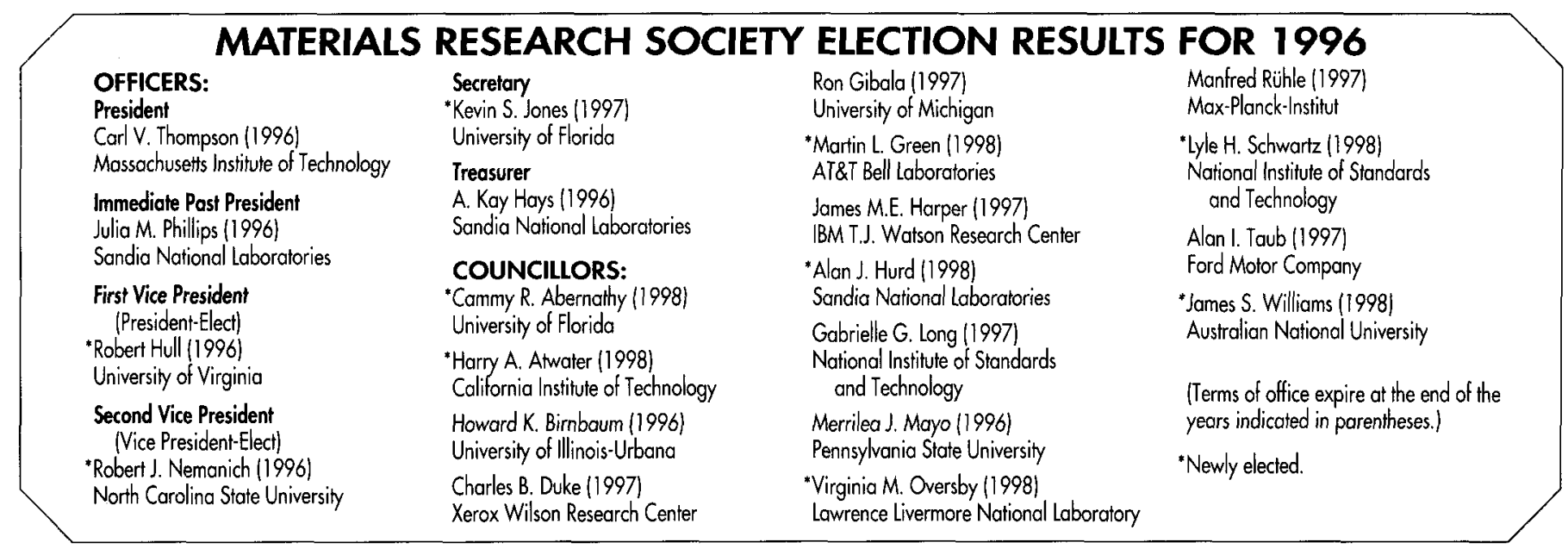

